\title{
EXCEPTIONAL SMOOTH BOL LOOPS
}

\section{LARISSA V. SBITNEVA}

Received 18 February 2001 and in revised form 8 August 2001

One new class of smooth Bol loops, exceptional Bol loops, is introduced and studied. The approach to the Campbell-Hausdorff formula is outlined. Bol-Bruck loops and Moufang loops are exceptional which justifies our consideration.

2000 Mathematics Subject Classification: 20N05.

1. Basic differential equations. We consider a $C^{3}$-smooth (and, consequently, $C^{\omega}$ smooth) right Bol loop $2=\langle Q, \cdot, \varepsilon\rangle$ with the defining identity $y \cdot[(b \cdot x) \cdot b]=[(y$. $b) \cdot x] \cdot b$ (right Bol identity), or, in another form, $R_{b} * R_{x} * R_{b}=R_{(b \cdot x) \cdot b}, R_{b} z \stackrel{\text { def }}{=} z \cdot b$. (Here $(f * g) y=(g \circ f) y=g(f(y))$.)

The differential equation of a right Bol loop is of the form

$$
\begin{gathered}
-A_{\sigma}^{\beta}(x) \frac{\partial(x \cdot y)^{\alpha}}{\partial x^{\beta}}+2 \Gamma_{\sigma}^{\beta}(y) \frac{\partial(x \cdot y)^{\alpha}}{\partial y^{\beta}}=A_{\sigma}^{\alpha}(x \cdot y), \\
A_{\tau}^{\mu}(z)=\left[\frac{\partial(z \cdot b)^{\mu}}{\partial b^{\tau}}\right]_{b=\varepsilon}, \quad a_{\tau}^{\mu}(z)=\left[\frac{\partial(b \cdot z)^{\mu}}{\partial b^{\tau}}\right]_{b=\varepsilon}, \\
\Gamma_{\tau}^{\mu}(z)=\frac{1}{2}\left[A_{\tau}^{\mu}(z)+a_{\tau}^{\mu}(z)\right] .
\end{gathered}
$$

See [1, Chapter 5].

According to general theory, the conditions of integrability for (1.1) are

$$
\left[A_{\alpha},\left[A_{\beta}, A_{\gamma}\right]\right]=\rho_{\alpha, \beta \gamma}^{v} A_{\nu}, \quad A_{\nu}(x)=A_{\nu}^{\tau}(x) \frac{\partial}{\partial x^{\tau}}, \quad \rho_{\alpha, \beta \gamma}^{v}=\text { const., }
$$

where $A_{v}$ is the so-called basic left fundamental vector fields. Evidently $A_{\mu}^{\nu}(\varepsilon)=\delta_{\mu}^{\nu}$.

Since a $C^{3}$-smooth right Bol loop is right monoalternative, that is, $(a \cdot t b) \cdot u b=$ $a \cdot(t+u) b$, where $t b=\operatorname{Exp}$

$$
\left[(\operatorname{Exp} t \zeta)^{\alpha}\right]^{\prime}=A_{\lambda}^{\alpha}(\operatorname{Exp} t \zeta) \zeta^{\lambda},\left.\quad(\operatorname{Exp} t \zeta)\right|_{t=0}=\varepsilon
$$

we get the differential equation

$$
\left[(a \cdot t b)^{\alpha}\right]^{\prime}=A_{\beta}^{\alpha}(a \cdot t b)\left(\operatorname{Exp}^{-1} b\right)^{\beta}, a \cdot 0 b=a,
$$

(see [1, Propositions 4.12, 4.22]), allowing to restore the law of composition $a \cdot b=$ $\psi(a, b, 1)$, where $\psi(a, b, t)=a \cdot t b$ is the solution of (1.4) with the initial condition $\psi(a, b, 0)=a$. 
2. Conditions of integrability. Now we introduce

$$
\left[A_{\alpha}, A_{\beta}\right](x)=c_{\alpha \beta}^{\gamma}(x) A_{\gamma}(x), \quad c_{\beta}^{\gamma}(x)=\left(\operatorname{Exp}^{-1} x\right)^{\alpha} c_{\alpha \beta}^{\gamma}(x) .
$$

Then (1.2) means $A_{\alpha}(x)\left(c_{\beta \gamma}^{v}(x) A_{v}(x)\right)-c_{\beta \gamma}^{v}(x) A_{v}(x) A_{\alpha}(x)=\rho_{\alpha, \beta \gamma}^{\mu} A_{\mu}(x)$, or

$$
A_{\alpha}^{\sigma}(x) \frac{\partial c_{\beta \gamma}^{\mu}(x)}{\partial x^{\sigma}}+c_{\beta \gamma}^{\nu}(x) c_{\alpha \nu}^{\mu}(x)=\rho_{\alpha, \beta \gamma}^{\mu}
$$

Taking $x=t b$ and contracting both parts with $\left(\operatorname{Exp}^{-1} b\right)^{\alpha},\left(\operatorname{Exp}^{-1} b\right)^{\beta}$, in normal coordinates $\left(\left(\operatorname{Exp}^{-1} b\right)^{v}=b^{v}\right)$, we get

$$
b^{\alpha} A_{\alpha}^{\sigma}(t b) \frac{\partial\left\{b^{\beta} c_{\beta \gamma}^{\mu}(t b)\right\}}{\partial(t b)^{\sigma}}+b^{\beta} c_{\beta \gamma}^{v}(t b) b^{\alpha} c_{\alpha \nu}^{\mu}(t b)=\rho_{\alpha, \beta \gamma}^{\mu} b^{\alpha} b^{\beta} .
$$

According to (1.4), $\left(\operatorname{Exp}^{-1} b\right)^{\alpha} A_{\alpha}^{\sigma}(t b)=d(t b)^{\sigma} / d t$, and, instead of (2.3), we have

$$
\begin{aligned}
\left(c_{\gamma}^{\mu}\right)^{\prime}+c_{\nu}^{\mu} c_{\gamma}^{\nu} & =\rho_{\gamma}^{\mu},\left.\quad c_{\nu}^{\mu}\right|_{t=0}=c_{0}^{\mu}, \\
c_{\gamma}^{\mu}=b^{\beta} c_{\beta \gamma}^{\mu}(t b), \quad \rho_{\gamma}^{\mu} & =b^{\alpha} b^{\beta} \rho_{\alpha, \beta \gamma}^{\mu}, \quad c_{\circ}^{\mu}=b^{\beta} c_{\beta \gamma}^{\mu}(\varepsilon) .
\end{aligned}
$$

Thus $c_{\gamma}^{\mu}$ may be obtained as the unique solution of the differential equation (2.4).

3. Conditions on fundamental vector fields. In accordance with the general theory, [1, Chapter 4] , the fields $A_{v}(\nu=1, \ldots, r)$, or matrix $A_{v}^{\mu}(x)$, can be uniquely restored by $c_{\gamma}^{\mu}$ in normal coordinates $((\operatorname{Exp} b)=b)$. For this we should consider $B_{\sigma}^{v}(x)$, the inverse of $A_{v}^{\mu}(x)$, and, further, solve the equation

$$
\left[t B_{\mu}^{\lambda}(t b)\right]^{\prime}=\delta_{\mu}^{\lambda}-c_{\mu}^{\sigma}\left[t B_{\sigma}^{\lambda}(t b)\right],\left.\quad t B_{\mu}^{\lambda}\right|_{t=0}=0 .
$$

It gives us $B_{\mu}^{\lambda}(t b)$ in normal coordinates, as well as its inverse $A_{\sigma}^{\lambda}(t b)$.

4. Matrix form of differential equations. As a result, regarding

$$
\underset{\circ}{\bar{c}}=\left\|b^{\beta} c_{\beta \gamma}^{\mu}(\varepsilon)\right\|_{\mu, \gamma=1,2, \ldots, r}, \quad \bar{\rho}=\left\|\rho_{\alpha, \beta \gamma}^{\mu} b^{\alpha} b^{\beta}\right\|_{\mu, \gamma=1,2, \ldots, r}
$$

as given, we should solve, first, the equation

$$
\overline{\bar{c}}^{\prime}+\overline{\bar{c}}^{2}=\overline{\bar{\rho}},\left.\quad \overline{\bar{c}}\right|_{t=0}=\overline{\bar{c}}
$$

which is the matrix form of (2.4) and, further, solve the equation

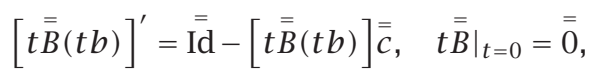

which is the matrix form of (3.1).

The solution of (4.2) may be presented as a series decomposition depending on $\overline{\bar{c}}$ and $\overline{\bar{\rho}}$, but the formulas are rather complicated, since $\underset{\mathrm{c}}{\overline{\bar{c}}}$ and $\overline{\bar{\rho}}$ do not commute in general. 
5. Bol loops of special type. If 2 is a Bol-Bruck loop, we have $\underset{\bar{c}}{\bar{c}}=0$, due to the automorphic inverse property $(x \cdot y)^{-1}=x^{-1} \cdot y^{-1}$, valid for any Bol-Bruck loop.

Indeed, differentiating the above relation by $x$ and $y$ at $x=y=\varepsilon$, we get $c_{\beta \gamma}^{\alpha}(\varepsilon)=0$ and, further, $\overline{\bar{c}}=\stackrel{\bar{\nu}}{0}$.

In the Moufang case we have, see [1, Chapter 6, (6.12)], $\overline{\bar{\rho}}=\overline{\bar{c}}^{2}$. Thus for Bol-Bruck and Moufang cases, in normal coordinates, we have

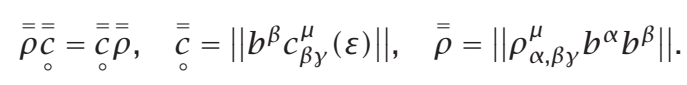

DEFINITION 5.1. A right Bol loop satisfying (5.1) is called exceptional.

REMARK 5.2. The question if the exceptional Bol loops may be described in the terms of identities depending on loop (or odule) operations only, is open.

REMARK 5.3. Of course, (5.1) may be rewritten as identity on $c_{\circ \gamma}^{\mu}(\varepsilon)$ and $\rho_{\alpha, \beta \gamma}^{\mu}$ which, together with the identities of a right Bol algebra, defines an exceptional Bol algebra.

6. Explicit form of structure functions. For an exceptional Bol algebra, due to (5.1), the solution of (4.2) is, evidently,

$$
\overline{\bar{c}}=\overline{\bar{\rho}}^{1 / 2} \tanh \left(\overline{\bar{\rho}}^{1 / 2} t+\overline{\bar{a}}\right), \quad \overline{\bar{a}}=\tanh ^{-1}\left(\overline{\bar{\rho}}^{-1 / 2} \underset{\mathrm{c}}{\bar{c}}\right)
$$

and may be expressed as

$$
\overline{\bar{c}}=\left[\overline{\bar{\rho}}^{1 / 2} \tanh \left(\overline{\bar{\rho}}^{1 / 2} t\right)+\overline{\bar{c}}\right]\left[\operatorname{Id}+\overline{\bar{c}}_{\circ}\left(\overline{\bar{\rho}}^{-1 / 2} \tanh \left(\overline{\bar{\rho}}^{1 / 2} t\right)\right)\right]^{-1} .
$$

Of course, (6.1) or (6.2) should be understood in the right way: there is no need for the existence of $\overline{\bar{\rho}}^{1 / 2}$, since the final result in the form of the series depends only on $\overline{\bar{\rho}}$.

7. Explicit form of basic vector fields. Now we solve (4.3), where $\overline{\bar{c}}$ is given by (6.1),

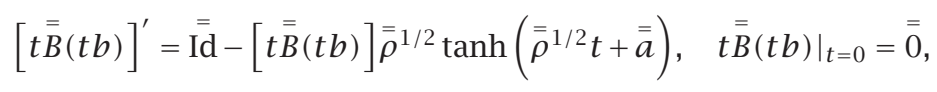

or

$$
\left[t \overline{\bar{B}}(t b) \cosh \left(\overline{\bar{\rho}}^{1 / 2} t+\overline{\bar{a}}\right)\right]^{\prime}=\cosh \left(\overline{\bar{\rho}}^{1 / 2} t+\overline{\bar{a}}\right),\left.\quad t \overline{\bar{B}}(t b)\right|_{t=0}=\overline{\overline{0}} .
$$

Thus

$$
t \stackrel{\bar{B}}{B}(t b) \cosh \left(\overline{\bar{\rho}}^{1 / 2} t+\overline{\bar{a}}\right)=\overline{\bar{\rho}}^{-1 / 2} \sinh \left(\overline{\bar{\rho}}^{1 / 2} t+\overline{\bar{a}}\right)+\overline{\bar{D}}
$$


Since $\left.[t \overline{\bar{B}}(t b)]\right|_{t=0}=\overline{\overline{0}}$, we get $\overline{\bar{D}}=-\overline{\bar{\rho}}^{-1 / 2} \sinh \overline{\bar{a}}$, and, at $t=1$, we have

$$
\stackrel{\bar{B}}{B}(b)=\overline{\bar{\rho}}^{-1 / 2} \frac{\left[\sinh \left(\overline{\bar{\rho}}^{1 / 2}+\overline{\bar{a}}\right)-\sinh \overline{\bar{a}}\right]}{\left[\cosh \left(\overline{\bar{\rho}}^{1 / 2}+\overline{\bar{a}}\right)\right]}, \quad \overline{\bar{a}}=\tanh ^{-1}\left(\overline{\bar{\rho}}^{-1 / 2} \underset{c}{c}\right) .
$$

Or, using the formulas of hyperbolic trigonometry, we get

$$
\overline{\bar{B}}(b)=\overline{\bar{\rho}}^{-1 / 2} \frac{\left(\cosh \overline{\bar{\rho}}^{1 / 2}-\mathrm{Id}\right) \tanh \overline{\bar{a}}+\sinh \overline{\bar{\rho}}^{1 / 2}}{\cosh \overline{\bar{\rho}}^{1 / 2}+\left(\sinh \overline{\bar{\rho}}^{1 / 2}\right) \tanh \overline{\bar{a}}},
$$

and, by (7.4),

$$
\overline{\bar{B}}(b)=\frac{\overline{\bar{\rho}}^{-1}\left(\cosh \overline{\bar{\rho}}^{1 / 2}-\mathrm{Id}\right) \underset{\circ}{\bar{c}}+\overline{\bar{\rho}}^{-1 / 2} \sinh \overline{\bar{\rho}}^{1 / 2}}{\cosh \overline{\bar{\rho}}^{1 / 2}+\left(\overline{\bar{\rho}}^{-1 / 2} \sinh \overline{\bar{\rho}}^{1 / 2}\right) \overline{\bar{c}}}, \quad \bar{\rho}_{\beta}^{\alpha}=\rho_{\mu, v \beta}^{\alpha} b^{\mu} b^{v} .
$$

As a result,

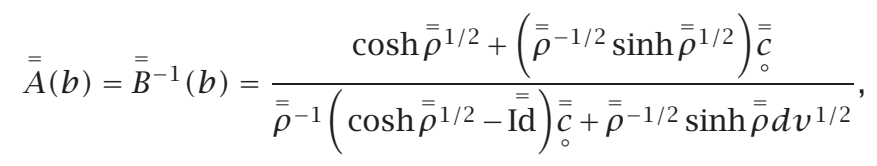

meaning that $\overline{\bar{A}}(b)=\stackrel{\bar{I}}{\mathrm{Id}}+\sum_{k=1}^{\infty} \overline{\bar{a}}_{k}(\overline{\bar{\rho}})^{k}$, where $\overline{\bar{a}}_{k}$ depends on $\underset{\bar{c}}{\bar{c}}$ only.

8. Analogue of the Campbell-Hausdroff decomposition. Now, using the differential equation (1.4) in normal coordinates (i.e., $\left.\left(\operatorname{Exp}^{-1} b\right)^{\mu}=b^{\mu}\right)$, we may obtain by successive differentiation with respect to $t$ :

$$
\frac{d^{k}(a \cdot t b)^{\alpha}}{d t^{k}}=\left\{A_{\sigma_{1}} A_{\sigma_{2}} \cdots A_{\sigma_{k}}(a \cdot t b)^{\alpha}\right\} b^{\sigma_{1}} b^{\sigma_{2}} \cdots b^{\sigma_{k}}
$$

As a result the Taylor decomposition

$$
(a \cdot b)^{\alpha}=a^{\alpha}+\sum_{m=1}^{\infty} \frac{1}{m !}\left(A_{\sigma_{1}} A_{\sigma_{2}} \cdots A_{\sigma_{m}} a\right)^{\alpha} b^{\sigma_{1}} b^{\sigma_{2}} \cdots b^{\sigma_{m}}
$$

is valid for an appropriate neighbourhood of $(0, \ldots, 0)$.

Taking $A_{\sigma}(a)=A_{\gamma}^{v}(a) \partial / \partial a^{v}$, where the matrix $A_{\gamma}^{v}(a)$ is defined by (7.7), we get the analogue of the Campbell-Hausdorff series for a smooth exceptional right Bol loop. Certain technical efforts would allow us to obtain an explicit expression of the coefficients $\left(A_{\sigma_{1}} A_{\sigma_{2}} \cdots A_{\sigma_{m}} a\right)^{\alpha}$ for an exceptional right Bol loop which would lead to the analogue of the Dynkin formula from the Lie group theory. 
ACKNOWLEDGMENT. This work has been written under the financial support of Mexican National Council of Science and Technology (Grant No. 32236-E).

\section{REFERENCES}

[1] L. V. Sabinin, Smooth Quasigroups and Loops, Kluwer Academic Publishers, Dordrecht, 1999.

LaRisSa V. SbitneVA: DePartment of Mathematics, QUintana Roo UniVersity, 77019 Chetumal, Quintana Roo, MeXico

E-mail address: 1arissa@servm.fc. uaem.mx 


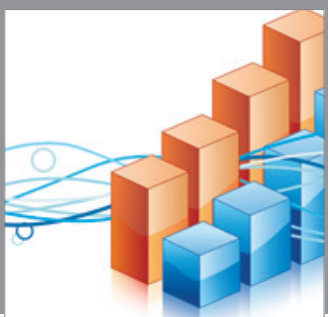

Advances in

Operations Research

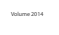

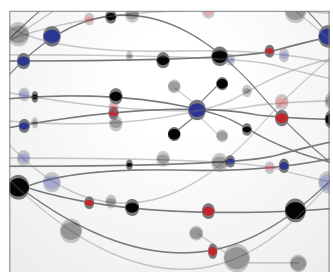

\section{The Scientific} World Journal
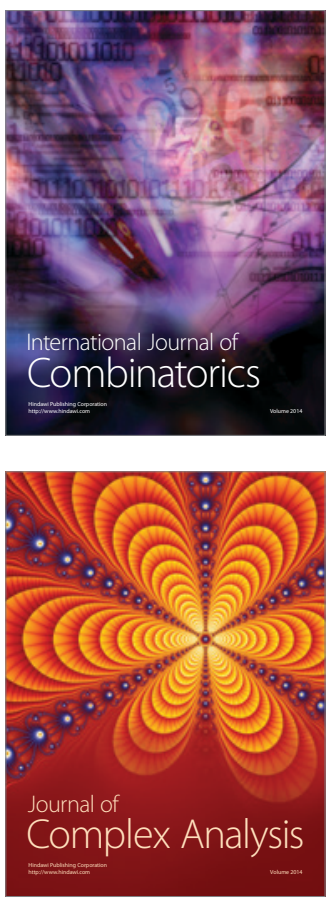

International Journal of

Mathematics and

Mathematical

Sciences
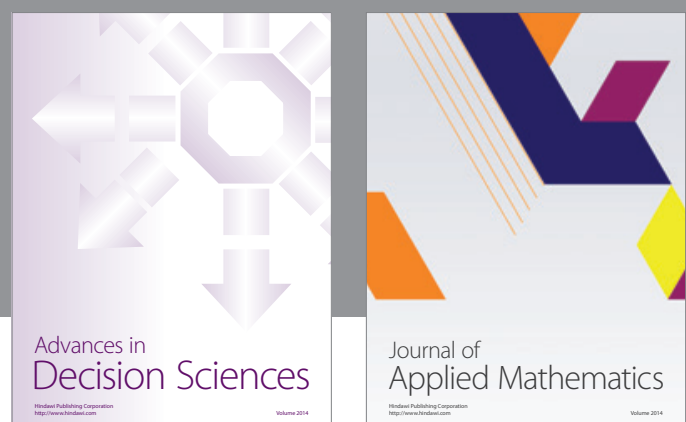

Journal of

Applied Mathematics
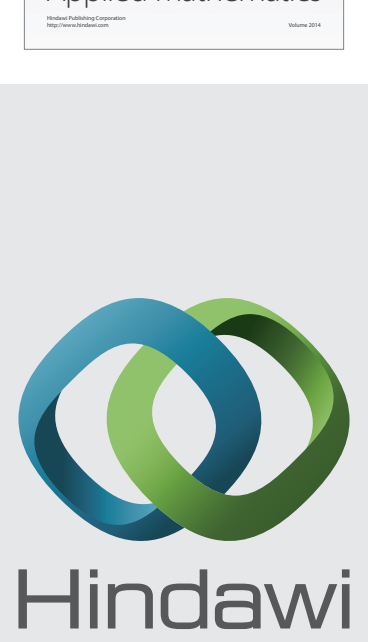

Submit your manuscripts at http://www.hindawi.com
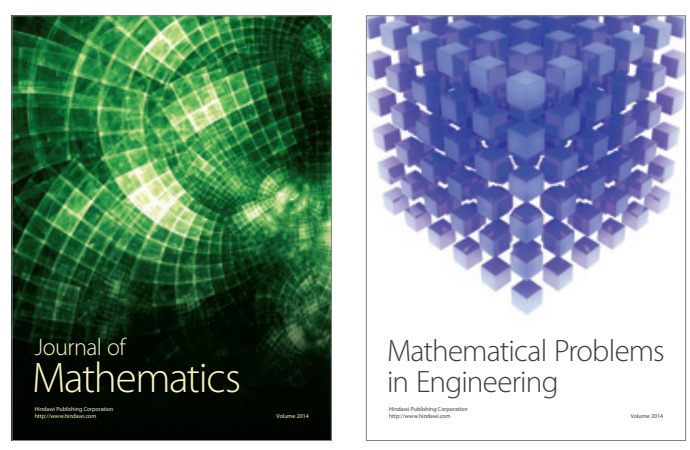

Mathematical Problems in Engineering
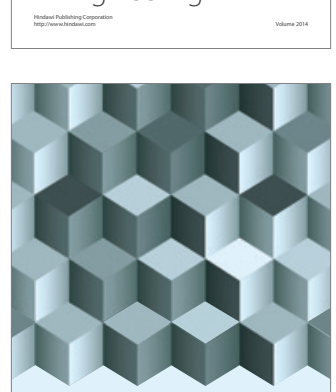

Journal of

Function Spaces
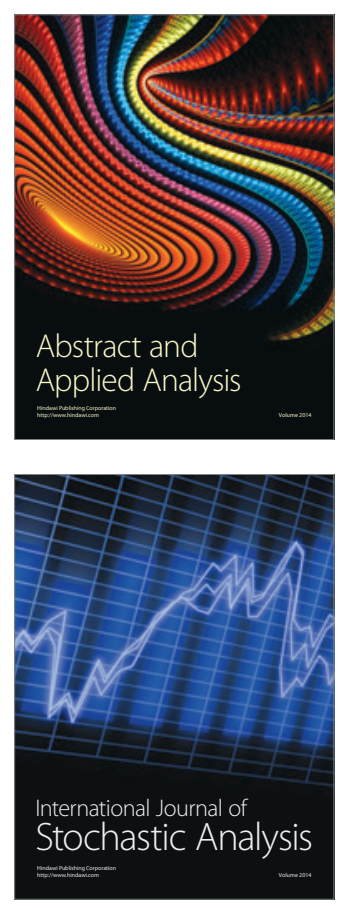

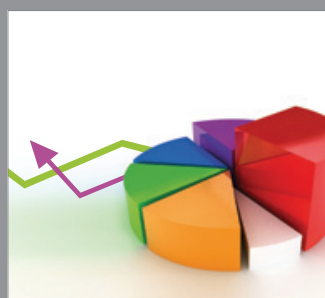

ournal of

Probability and Statistics

Promensencen
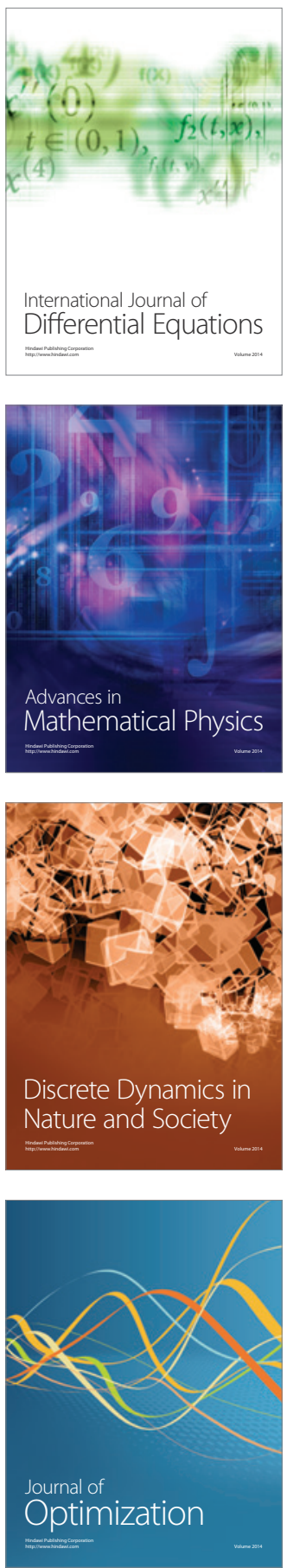